\title{
miRNAs involved in the development and differentiation of fertile and sterile flowers in Viburnum macrocephalum f. keteleeri
}

\author{
Weixing Li, Zhichong He, Li Zhang, Zhaogeng Lu, Jing Xu, Jiawen Cui, Li Wang and Biao Jin * (D)
}

\begin{abstract}
Background: Sterile and fertile flowers are important evolutionary developmental phenotypes in angiosperm flowers. The development of floral organs, critical in angiosperm reproduction, is regulated by microRNAs (miRNAs). However, the mechanisms underpinning the miRNA regulation of the differentiation and development of sterile and fertile flowers remain unclear.

Results: Here, based on investigations of the morphological differences between fertile and sterile flowers, we used high-throughput sequencing to characterize the miRNAs in the differentiated floral organs of Viburnum macrocephalum f. keteleeri. We identified 49 known miRNAs and 67 novel miRNAs by small RNA (sRNA) sequencing and bioinformatics analysis, and 17 of these known and novel miRNA precursors were validated by polymerase chain reaction (PCR) and Sanger sequencing. Furthermore, by comparing the sequencing results of two sRNA libraries, we found that 30 known and 39 novel miRNA sequences were differentially expressed, and 35 were upregulated and 34 downregulated in sterile compared with fertile flowers. Combined with their predicted targets, the potential roles of miRNAs in V. macrocephalum f. keteleeri flowers include involvement in floral organogenesis, cell proliferation, hormonal pathways, and stress responses. miRNA precursors and targets were further validated by quantitative real-time PCR (qRT-PCR). Specifically, miR156a-5p, miR156g, and miR156j expression levels were significantly higher in fertile flowers than in sterile flowers, while SPL genes displayed the opposite expression pattern. Considering that the targets of miR156 are predicted to be SPL genes, we propose that miR156 may be involved in the regulation of stamen development in $\mathrm{V}$. macrocephalum $\mathrm{f}$. keteleeri.

Conclusions: We identified miRNAs differentially expressed between fertile and sterile flowers in $V$. macrocephalum f. keteleeri and provided new insights into the important regulatory roles of miRNAs in the differentiation and development of fertile and sterile flowers.
\end{abstract}

Keywords: Viburnum macrocephalum f. keteleeri, miRNA profiling, Fertile and sterile flowers, Flower differentiation and development

\section{Background}

Flowers are crucial to the reproductive success and continuity of flowering plants over time [1,2]. In general, the floral meristem sequentially produces floral organs, including the sepals, petals, stamens, and carpels, which arise in concentric rings, or whorls [3]. However, flowers come in a variety of colors, structures, shapes, and sizes, which are the evolutionary consequences of selective

\footnotetext{
* Correspondence: bjin@yzu.edu.cn

College of Horticulture and Plant Protection, Yangzhou University, Yangzhou 225009, China
}

pressure by biotic and abiotic environmental factors $[4$, 5]. For example, flower size is an important characteristic shaped by insect pollinator behavior and selection, as different-sized flowers can result in different visiting frequencies, thus affecting reproductive success [6, 7]. Flowers can be classified as fertile or sterile according to their ability to reproduce sexually and produce available gametes. Fertile flowers generate normal stamens and pistils, while sterile flowers generally have abnormal reproductive structures, such as stamen, anther, and pollen abnormalities conferring sterility in males and stigma, pistil, and embryo sac abnormalities conferring sterility/ 
infertility in females. Although sterile flowers cannot produce fruits or seeds for the next generation, they play roles in improving pollination quality by attracting pollinators and reducing the reproductive costs associated with large floral displays, consequently enhancing reproductive success $[8,9]$. As a result, inflorescences composed of coexisting sterile and fertile flowers are very common in flowering plants, from grasses to trees.

Research in developmental genetics and genomics has facilitated studies of flower differentiation and development [10]. Many genes related to floral organ development have been identified. For example, the ABCDE model-related genes and MADS-box gene family are required for flower development in many angiosperm lineages [11]. MADS-box transcription factors and Zinc finger family proteins are involved in male fertile and sterile development, according to floral transcriptomic analyses [12]. Additionally, some genes, such as TCP and the GRFs family, regulate floral organ size [13].

MicroRNAs (miRNAs) are small, non-coding RNAs produced by Dicer-catalyzed excision from stem-loop precursors, which regulate gene expression at the posttranscriptional level by directing RNA cleavage or inhibiting translation of target transcripts [14]. Numerous studies have demonstrated the critical role of miRNAs in controlling flower development-related processes [15]. Some miRNAs, such as miR156 and miR172, have been found to control genes that function in flower transition [16]. Some evolutionarily conserved miRNAs can regulate the development of floral organs, including the growth and differentiation of sepals, petals, anthers, and carpels [17]. For example, miR319a is critical for petal growth and development in Arabidopsis through its targeting of TCP4, and miR159, with its target MYB, can affect anther development $[18,19]$. These findings suggest that miRNAs have diverse biological functions in flower development. However, the regulatory roles of miRNAs in fertile and sterile flower differentiation and development are still unclear.
Viburnum macrocephalum f. keteleeri, a Chinese wild shrub in the Adoxaceae family, has two kinds of flowers in an inflorescence [8]. The type distributed in the internal position of the inflorescence is fertile, whereas the sterile flowers are distributed around the exterior (Fig. 1). Fertile flowers are morphologically smaller than sterile flowers, but the number of fertile flowers is much greater than that of sterile flowers. In particular, these two kinds of flowers are produced from a single inflorescence, and the sterile flowers evolve from flowers that were initially fertile [8]. Therefore, $V$. macrocephalum $\mathrm{f}$. keteleeri is an ideal candidate species for investigating the differentiation of fertile and sterile flowers in the same genetic background. To determine the miRNAs regulating the development of fertile and sterile flowers, we used high-throughput sequencing and detected more than 11 million reads for each type of flower. We identified known and novel miRNAs in the flower organs of this species and investigated the targets of these miRNAs to explore the potential functions of the miRNAs. We analyzed the dynamic expression patterns of the miRNAs and their putative targets using our transcriptomic data (NCBI SRA [SRP076665] and GEO [GSE83429]). Quantitative real-time polymerase chain reaction (qRT-PCR) was used to validate the dynamic expression patterns of the miRNAs and their putative targets. The results of this study contribute to our understanding of the regulatory roles of miRNAs in the development of fertile and sterile flowers.

\section{Methods}

\section{Plant materials}

We used adult $V$. macrocephalum f. keteleeri plants grown at Yangzhou University, Jiangsu, Eastern China $\left(32^{\circ} 39^{\prime} \mathrm{N}, 119^{\circ} 43^{\prime} \mathrm{E}\right)$. We observed and digitally recorded flowers at different developmental stages every week from March to May. Fertile and sterile flowers were collected from three phases of floral development: early (ES; Fig. 1a), middle (MS; Fig. 1b), and later (LS; Fig. 1c) stages of flower induction and the flowering process.
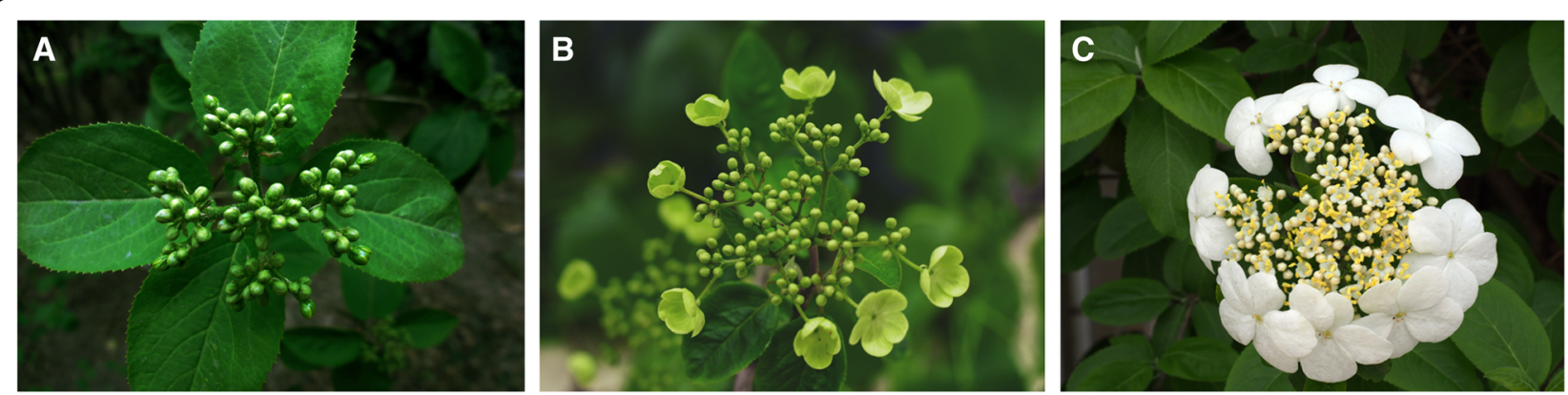

Fig. 1 Flowers of Viburnum macrocephalum f. keteleeri at three stages. a Flowers in early stage (ES). b Flowers in middle stage (MS). c Flowers in late stage (LS) 
Sufficient samples were immediately frozen in liquid nitrogen and stored at $-80{ }^{\circ} \mathrm{C}$ for RNA extraction, and some fresh tissues were also collected for morphological observations. Fertile and sterile flowers from the MS (the period of rapid differential growth; Fig. 1b) were selected for the construction of small RNA (sRNA) libraries.

\section{Morphological observations of flower petals}

For scanning electron microscope observation, flower petals collected at different stages were fixed in $2.5 \%$ glutaraldehyde (in $0.1 \mathrm{M}$ phosphate buffer, $\mathrm{pH}$ 7.2) for $2 \mathrm{~h}$ at $4{ }^{\circ} \mathrm{C}$. They were then rinsed three times in $0.1 \mathrm{M}$ phosphate buffer (15 min per rinse), dehydrated in a graded ethanol series $(30,50,70,80,90,95$, and 100\%; $15 \mathrm{~min}$ each), and dried by automatic critical point drying (Leica, Wetzlar, Germany). After sputter coating (Bal-Tec, Balzers, Switzerland), the samples were examined, imaged, and measured by scanning electron microscopy (SEM) (Hitachi High-Technologies Corporation, Tokyo, Japan) at $15.0 \mathrm{kV}$ [20]. Petal cell size measurements under SEM were based on three samples selected randomly at each stage. Averages and standard deviations were calculated using Microsoft Office Excel 2003. The surface area, size, and number of petal cells were calculated as follows: 1 ) Image J (1.48) software was used to measure the surface areas of the flower petals. 2) Measurements of petal cell size using SEM were conducted in three samples selected randomly at each stage of floral development. More than 10 different positions on each petal sample were photographed; thus, at least 30 replicates in each fertile and sterile flower were used to calculate the average cell size. 3) Using the results from 1) and 2), we estimated the number of surface cells on flower petals at each floral stage as the surface area of the flower petal (the result from 1) divided by the petal cell size (the result from 2).

For semi-thin section observations, the sterile and fertile flowers were separately cut from buds to full-bloom stage flowers using a razor blade. Each sample was prefixed in $2.5 \%(\mathrm{v} / \mathrm{v})$ glutaraldehyde (in $0.1 \mathrm{~mol} / \mathrm{L}$ phosphate buffer, $\mathrm{pH} 7.2)$ at $4{ }^{\circ} \mathrm{C}$ for $2 \mathrm{~h}$. The samples were washed three times in $0.1 \mathrm{M}$ phosphate buffer ( $\mathrm{pH} 7.2$, $10 \mathrm{~min}$ per rinse), dehydrated in an ethanol series (30, 50, 70, 80, 90, and 95\%; 30 min for each step), and then washed three times for $30 \mathrm{~min}$ with 100\% ethanol. Then, the ethanol/propylene oxide ratios were adjusted to $7: 3$, $5: 5$, and $3: 7$ by adding the oxide proportion. After the samples were treated three times with pure propylene oxide (10 min each rinse), the samples were infiltrated gradually with spurr resin [21]. Then they were agitated in an oscillator for $12 \mathrm{~h}$. Finally, resin masses were acquired by oven drying. For ultrastructural observations, 70-nm thick sections were cut with a Leica EM UC6 ultramicrotome (Leica) and stained with Toluidine blue. The cells were observed and photographed under the Primo Star biological microscope (ZEISS, Oberkochen, Germany).

\section{sRNA sequencing}

Two sRNA libraries, from fertile and sterile flowers, were constructed and sequenced by Novogene Biotechnology Corporation (Beijing, China) using the Illumina Genome Analyzer. Total RNA was isolated from $V$. macrocephalum $\mathrm{f}$. keteleeri sterile flowers (VMS) and fertile flowers (VMF) using the Mini BEST Plant RNA Extraction Kit (TaKaRa, Dalian, China) and treated with genomic DNA (gDNA) Eraser (TaKaRa) to eliminate any DNA contamination. RNA degradation, contamination, purity, and integrity were all measured to ensure high quality. RNA degradation and contamination were monitored on $1 \%$ agarose gels. RNA purity was assessed using the NanoPhotometer ${ }^{\circledR}$ spectrophotometer (IMPLEN, CA, USA), and the RNA concentration was measured using the Qubit ${ }^{\oplus}$ RNA Assay Kit and the Qubit $^{\top}$ 2.0 Fluorometer (Life Technologies, CA, USA). RNA integrity was assessed using the RNA Nano 6000 Assay Kit of the Agilent Bioanalyzer 2100 system (Agilent Technologies, CA, USA). Sequencing libraries were generated using the NEBNext ${ }^{\circ}$ Multiplex Small RNA Library Prep Set for Illumina (New England Biolabs, Inc., Ipswich, MA, USA) following the manufacturer's recommendations, and index codes were added to attribute sequences to each sample.

Identification and prediction of known and novel miRNAs After quality control and removing tags from these sources, mapped sRNA tags were used to look for known and novel miRNAs. The sRNA annotation process was performed as follows: 1) The sRNA tags were first mapped to the reference sequence (our transcriptome data sets NCBI SRA [SRP076665] and GEO [GSE83429]) using Bowtie [22, 23], without allowing any mismatches, to analyze their expression and distribution relative to the reference sequence. 2) Next, the mapped sRNA tags were used to search for known miRNAs using a modification of the miRDeep2 program (with miRBase used as the reference). 3) The mapped sRNA tags were also mapped to Rfam, RepeatMasker, to annotate the tags and remove those originating from proteincoding genes, repeat sequences, ribosomal RNAs (rRNAs), transfer RNAs (tRNAs), small nuclear RNAs (snRNAs), and small nucleolar RNAs (snoRNAs). 4) The remaining unannotated tags were used to predict novel miRNAs by exploring secondary structures, Dicer cleavage sites, and minimum free energies using the miREvo and miRDeep2 software packages [24, 25]. Novo Custom scripts (Beijing Novo Gene Genomics Institute, China) were used to obtain the miRNA counts; they were also used to obtain the base bias at the first position of the 
identified miRNAs of certain lengths and, subsequently, at each position of all identified miRNAs.

\section{Expression analysis of miRNAs and prediction of miRNA targets}

MiRNA expression levels were estimated as transcripts per million (TPM) using the following calculation: normalized expression $=$ mapped $\mathrm{read}$ count $/$ total mapped reads $\times 1,000,000$. Differential expression analysis of two flowering phases was performed using the DESeq $R$ package (1.8.3). miRNAs that had change ratios of greater than 2 or less than 0.5 (Fold change Log2 $>1$ or $<-1$ ) and $p<0.01$ were set as the default thresholds for significant differential expression [26].

We used the web-based psRNATarget program (http:// plantgrn.noble.org/psRNATarget/) and psRobot_tar in psRobot to identify putative targets for known and novel miRNAs [27]. We used psRobot software to evaluate all predicted target genes using a previously defined scoring system. Genes with a score $<3$ were considered miRNA targets [28].

\section{Cloning and sequencing of pre-miRNA sequences}

Total RNA was isolated from VMS and VMF at MS as described above. cDNAs were synthesized from $2 \mu \mathrm{g}$ of purified total RNA in $40 \mu \mathrm{l}$ reactions using the PrimeScript $^{\text {TM }} 1$ st Strand cDNA Synthesis Kit (TaKaRa) according to the manufacturer's protocol. We designed 28 pairs of primers for $V$. macrocephalum $\mathrm{f}$. keteleeri precursor miRNA sequences (Additional file 1). PCR amplifications were carried out, using the following thermal cycling conditions: $94{ }^{\circ} \mathrm{C}$ for $5 \mathrm{~min}, 35$ cycles at $94{ }^{\circ} \mathrm{C}$ for $30 \mathrm{~s}, 55{ }^{\circ} \mathrm{C}$ or $60{ }^{\circ} \mathrm{C}$ for $15 \mathrm{~s}$, and $72{ }^{\circ} \mathrm{C}$ for $50 \mathrm{~s}$. Amplification products were separated on a $2 \%$ agarose gel with GelRed nucleic acid staining. Gel-purified PCR fragments were subcloned into the T5-simple Vector system (TransGen, Beijing, China) and sequenced.

\section{qRT-PCR validation of miRNAs and their target genes}

The RNA solution $(10 \mu \mathrm{l})$, after removal of DNA contamination, was subjected to reverse transcriptase reactions with PrimeScript ${ }^{\mathrm{Tm}}$ Reverse Transcriptase Reagent Kit with gDNA Eraser (Perfect Real Time) (TaKaRa) in accordance with the manufacturer's protocol. Specific primers for 35 miRNAs and 6 target genes were designed using Primer Premier 5.0 (Additional file 1). qRTPCR was performed using the SYBR Green PCR Master Mix (TaKaRa) on a CFX96 Detection System (Bio-Rad, Hercules, CA, USA). Briefly, the $25 \mu \mathrm{l}$ PCR reaction contained no more than 100 ng cDNA, $12.5 \mu \mathrm{l}$ SYBR Premix Dimer Eraser $(2 \times)$, and $0.3 \mu \mathrm{M}$ of each primer. The reactions were mixed and incubated at $95{ }^{\circ} \mathrm{C}$ for $30 \mathrm{~s}$, followed by 40 cycles of $95{ }^{\circ} \mathrm{C}$ for $5 \mathrm{~s}, 55^{\circ} \mathrm{C}$ for $30 \mathrm{~s}$, and $72{ }^{\circ} \mathrm{C}$ for $30 \mathrm{~s}$. The expression levels of the miRNAs and target genes were normalized to those of the internal controls U6 [29] and actin (NCBI, XM_002282480) respectively. The relative expression levels were analyzed using the $2^{-\Delta \Delta C T}$ method [30]. Ct represents the threshold cycle.

\section{Results \\ Morphological and structural observations of floral organs}

Flowers were observed from late March to late April with respect to the differentiation and development into fertile and sterile flowers. The morphological changes of fertile and sterile flowers at different stages were compared by recording images with a digital camera. In the ES (March 20-30), fertile and sterile flower petals were green; in the MS (March 30 to April 15), the color gradually changed from green to white; and, at the LS (April 15-30), the color was completely white (Fig. 2a). Obviously, the petal area of sterile flowers increased significantly during the developmental process, and it did so to a much greater extent than did that of the fertile flowers. For example, in the ES, the petal areas of fertile and sterile flowers were similar, about $30 \mathrm{~mm}^{2}$. However, after the ES, sterile flowers expanded rapidly; in the MS, the petal area of the sterile flowers increased from $325.331 \mathrm{~mm}^{2}$ to $1028.181 \mathrm{~mm}^{2}$, whereas the fertile flower petal area increased by only $14 \mathrm{~mm}^{2}$. At the LS, the petal area of sterile flowers reached about $1600 \mathrm{~mm}^{2}$ and was more than 20 times that of fertile flowers (Fig. 2d).

SEM photographs displayed the cellular morphology of fertile and sterile petals in the ES, MS, and LS (Fig. $2 b$ ). In the ES, petal cells were loosely packed (Fig. 2b, a and d). The flat surface area of a sterile flower petal cell is about $154 \mu \mathrm{m}^{2}$, and that of fertile petals is about $283 \mu \mathrm{m}^{2}$ (Fig. 2e). In the MS, cells of both flowers were arranged closely, and the intercellular space was small (Fig. 2b-e). The cellular area of sterile flowers was a little more than that of fertile flowers, at $542 \mu \mathrm{m}^{2}$ and $430 \mu \mathrm{m}^{2}$, respectively (Fig. 2e). In the LS, cells were arranged compactly (Fig. $2 \mathrm{~b}-\mathrm{f}$ ). The cellular area of sterile flowers increased to $873 \mu \mathrm{m}^{2}$, with many dividing cells, about twice the size of fertile flowers (Fig. 2e). In addition to the petal area, we also estimated the number of cells on the petal surfaces of fertile and sterile flowers at each stage. The cell count increased from $30 \times 10^{4}$ to $140 \times 10^{4}$ from the ES to LS in sterile flowers, whereas it remained constant, at approximately $10 \times 10^{4}$, in fertile flowers (Fig. 2f).

The anatomical features of VMS and VMF are shown in Fig. 2c. There were no stamens, or only thin and short stamens, in VMS (Fig. 2c, a and b). In contrast, the stamens of VMF had long filaments and normal anthers with pollen grains and anther septa (Fig. 2c-e). 


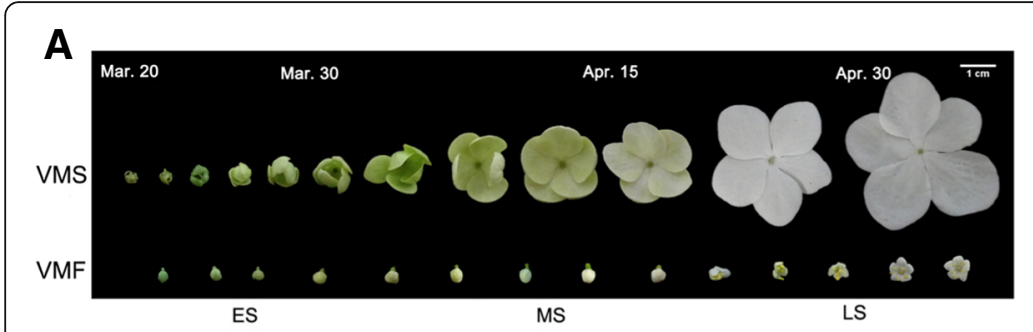

\section{B}
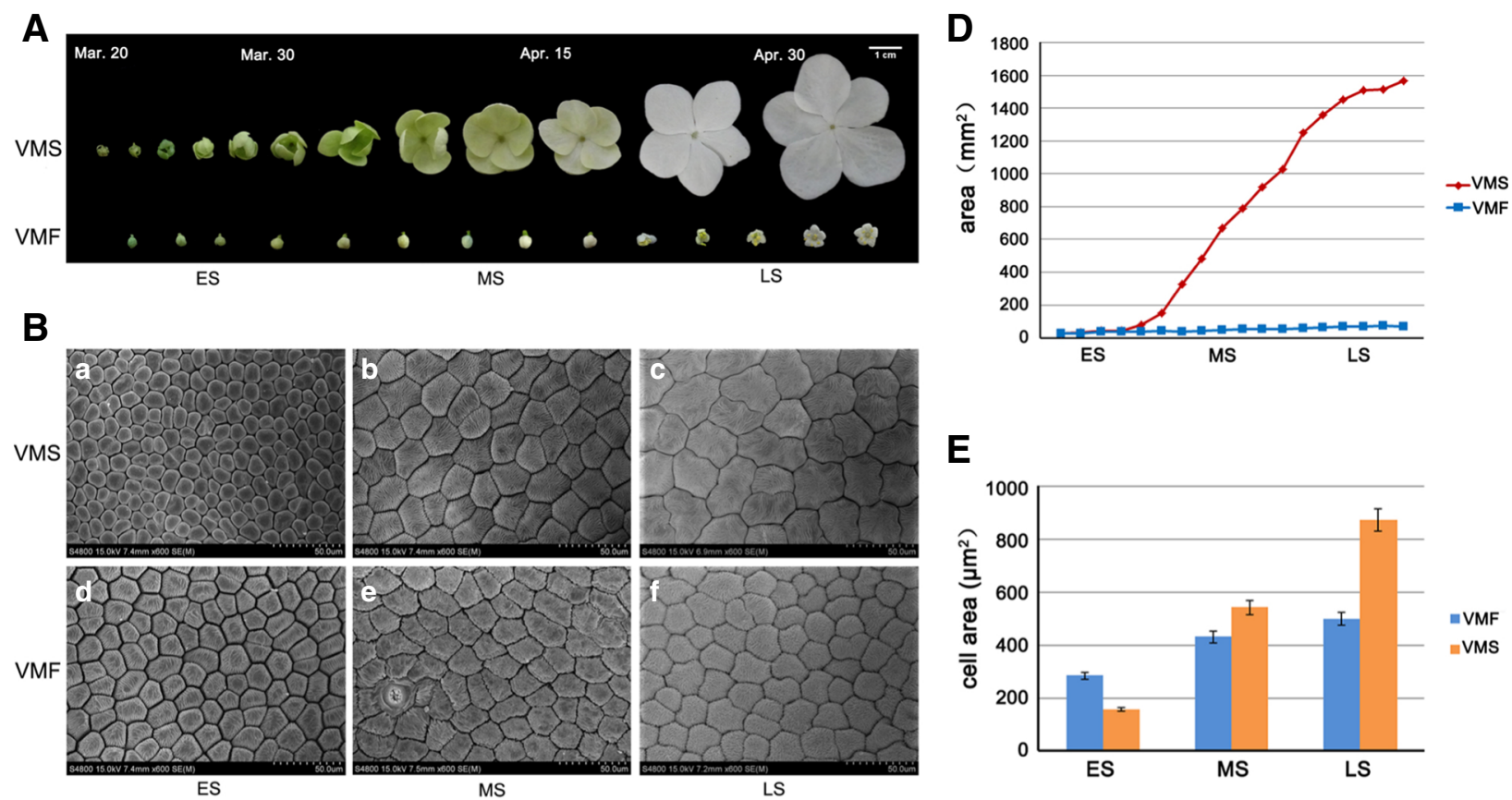

E

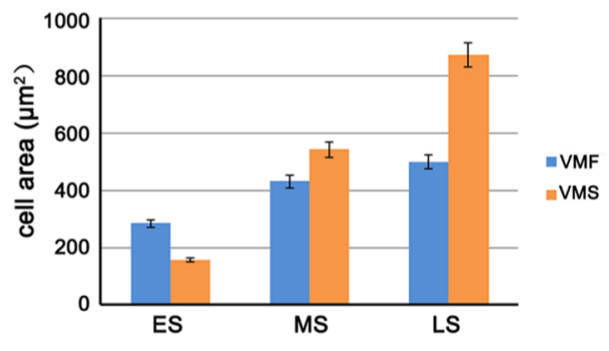

C
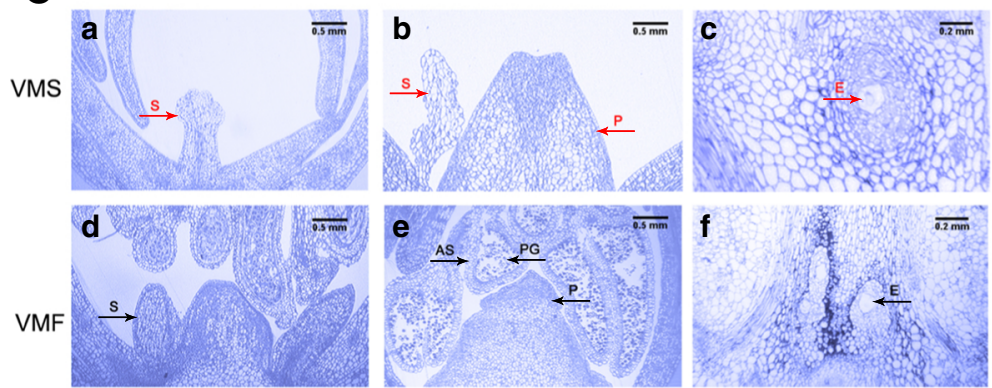

$\mathbf{F}$

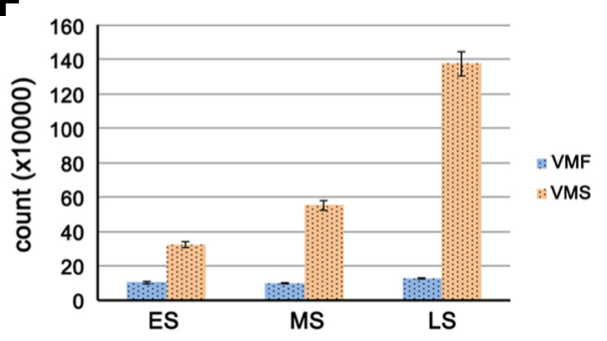

Fig. 2 Morphological and structural observations of floral organs. a Fertile (VMF) and sterile (VMS) flowers of V. macrocephalum f. keteleeri observed by digital camera from early to late April. Scale bar: $1 \mathrm{~cm}$. b Cellular morphology of VMF (d-e) and VMS (a-c) at three different developmental stages. Scale bars: 50 mm. c Anatomical features of VMF (d-e) and VMS (a-c). Red arrows indicate the short stamens (a), abnormal stamens (b), and the abnormal embryo sac with cavity in the ovary (c) of VMS. Black arrows indicate the normal stamens (d), stamens with anthers (e), and normal embryo sacs (f) of VMF. S: stamen, E: embryo sac, P: pistil, PG: pollen grain, AS: anther septa. Scale bars in a, b, d, and e: $0.5 \mathrm{~mm}$; scale bars in c and f: $0.2 \mathrm{~mm}$. $\mathbf{d}$ Petal areas of VMF and VMS. e Cell counts of VMF and VMS. $\mathbf{f}$ Cell areas of VMF and VMS

Additionally, relative to the normal pistils of fertile flowers, the pistils in sterile flowers lacked normal embryo sacs (Fig. 2c-f). These results indicate that sterile flowers have remarkably low fertility, with degraded stamens and pistils, whereas the stamens and pistils are normal, displaying healthy fertility, in fertile flowers of $V$. macrocephalum f. keteleeri.

\section{An overview of high-throughput sequencing datasets in fertile and sterile flowers}

To obtain a comprehensive profile of the sRNAs involved in $V$. macrocephalum flowers, two sRNA libraries (VMF and VMS) were constructed. A total of 11,447,459 and $12,365,813$ raw sRNA reads were generated,
Table 1 Summary of sRNA sequencing statistics

\begin{tabular}{lll}
\hline Type & $\begin{array}{l}\text { VMF Count } \\
\text { (percent) }\end{array}$ & $\begin{array}{l}\text { VMS Count } \\
\text { (percent) }\end{array}$ \\
\hline Total reads & $\begin{array}{l}11,447,459 \\
(100.00 \%)\end{array}$ & $\begin{array}{l}12,365,813 \\
(100.00 \%)\end{array}$ \\
N\% > 10\% & $0(0.00 \%)$ & $2(0.00 \%)$ \\
low quality & $7268(0.06 \%)$ & $6973(0.06 \%)$ \\
3_adapter_null or & $241,136(2.11 \%)$ & $491,043(3.97 \%)$ \\
insert_null & & \\
5_adapter_contamine & $36,161(0.32 \%)$ & $36,161(0.32 \%)$ \\
with poly A/T/G/C & $30,903(0.27 \%)$ & $43,226(0.35 \%)$ \\
Clean reads & $11,131,991(97.24 \%)$ & $11,803,832(95.46 \%)$ \\
\hline
\end{tabular}


respectively (Table 1). After removing low-quality reads, adapter sequences, poly $\mathrm{N}$ sequences, sequences of $<18$ nucleotides (nt), and other artifacts, 7,562,443 and 7,373,905 high-quality clean reads between $18 \mathrm{nt}$ and $30 \mathrm{nt}$ in length, respectively, remained for further analyses (Additional file 2).

We then summarized the length distribution of the sRNA libraries of fertile and sterile flowers. The size distribution of sequenced sRNAs was similar in both samples. The majority of clean sRNA reads were 21-24 nt in both libraries. The $24 \mathrm{nt}$ sRNAs were the most abundant, at approximately 3,028,365 (40.04\%) and 3,480,318 $(47.20 \%)$ in fertile and sterile flower libraries, respectively (Fig. 3). Additionally, 21, 22, and 23 nt sRNAs were more common than those of any other length besides 24 nt.

A total of 3,327,774 (44\%) and 2,811,600 (38.13\%) reads from fertile and sterile flowers, respectively, were mapped to non-coding sRNA database (Additional file 3). The mapped sRNA sequences were clustered into several RNA classes, such as known miRNA, rRNA, tRNA, snRNA, snoRNA, repeats, and TAS (Additional file 4). Most of these sRNAs ( $89.85 \%$ and $90.40 \%$, respectively) were clustered in the uncharacterized group. The second most abundant group (5.46\% and $4.76 \%$, respectively) was rRNA. 37,648 and 47,191 sequences were known miRNAs, at $1.13 \%$ and $1.68 \%$ in VMF and VMS, respectively. Additionally, 20,197 and 24,478 of the unmapped sRNA sequences were identified as novel miRNA candidates in VMF and VMS, respectively (Table 2).

\section{Identification of known and novel miRNAs}

We searched our results against miRBase 21.0 to annotate known miRNAs in $V$. macrocephalum f. keteleeri. Ultimately, we identified 226 and 199 unique sRNAs among the known miRNAs and 514 and 513 sRNAs among the novel miRNAs in fertile and sterile flowers, respectively (Table 2). After screening, 49 mature known miRNAs were identified. All precursors of mature miRNAs can adopt hairpin

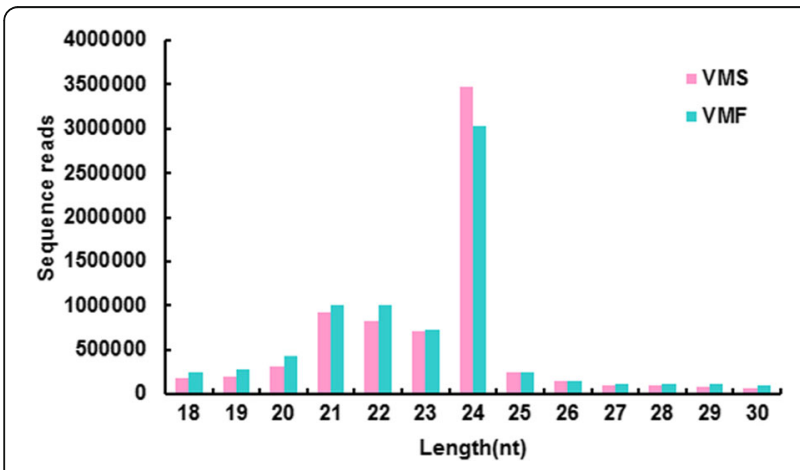

Fig. 3 Length distribution of small RNAs (sRNAs) from sterile and fertile flowers
Table 2 Summary of mapped mature and hairpin structures in known and novel miRNAs

\begin{tabular}{|c|c|c|c|c|c|c|}
\hline \multirow[t]{2}{*}{ Types } & \multicolumn{3}{|l|}{ Known } & \multicolumn{3}{|l|}{ Novel } \\
\hline & Total & VMF & VMS & Total & VMF & VMS \\
\hline Mapped total sRNA & 84,839 & 37,648 & 47,191 & 44,675 & 20,197 & 24,478 \\
\hline Mapped uniq sRNA & 425 & 226 & 199 & 1027 & 514 & 513 \\
\hline Mapped hairpin & 61 & 54 & 56 & 73 & 71 & 70 \\
\hline Mapped mature & 49 & 42 & 45 & 67 & 59 & 64 \\
\hline
\end{tabular}

structures resembling the fold-back structure of a miRNA precursor (Additional file 5). Analysis of nucleotide bias was performed on known miRNAs. The analysis showed that the first position of miRNAs of 18 to $24 \mathrm{nt}$ in length was occupied by uracil (U) over $80 \%$ of the time (Additional file 6). At the first and second positions, the percentage of $U$ averaged $97.73 \%$ and $80.80 \%$, respectively. The percentage of cytosine $(C)$ was the most increased at the last several base pair positions of the miRNAs compared with at the front positions.

A total of 24 miRNA families were identified among these known miRNAs (Fig. 4a). The largest family was miR156 and miR396, with four members, followed by miR159, miR167, miR169, miR172, and miR390, with three members; however, most miRNA families possessed only one family member (e.g., miR160, miR165, miR408). The abundance of known miRNAs varied greatly. miR159 had 25,054 and 42,454 reads in VMF and VMS, respectively, and was the most abundant. miR162, miR166, miR319, miR396, and miR403 all had abundance reads of more than a thousand, whereas many miRNAs (e.g., miR169, miR2111, miR397, miR398) were sequenced too infrequently to be distinguishable in the histogram (Fig. 4b; Additional file 7).

In addition to the known miRNAs, we identified novel miRNAs in flowers. According to the criteria for annotating novel miRNAs, a characteristic stem-loop precursor is a prerequisite [31]. A total of 73 novel miRNA precursors were predicted from 1027 unique sRNAs using miREvo and miRDeep2. The length of novel miRNA precursors ranged from 38 to $300 \mathrm{nt}$, with an average of $116 \mathrm{nt}$, and the MFE values obtained for these precursors ranged from $-12 \mathrm{kcal} / \mathrm{mol}$ to $-101.5 \mathrm{kcal} / \mathrm{mol}$, with an average of $-46.8 \mathrm{kcal} / \mathrm{mol}$ (Additional file 8 ). A total of 67 candidate novel miRNAs with clear precursors containing a stemloop secondary structure were identified (Fig. 5a; Additional file 8). The complementary miRNA* sequences for each candidate novel miRNA were also detected, although most were present at lower expression levels than their corresponding miRNAs (Additional file 9).

At the same time, custom scripts were used to analyze the nucleotide bias at the first position of miRNAs of certain lengths; this information was subsequently obtained for each position of all identified miRNAs. The 
A

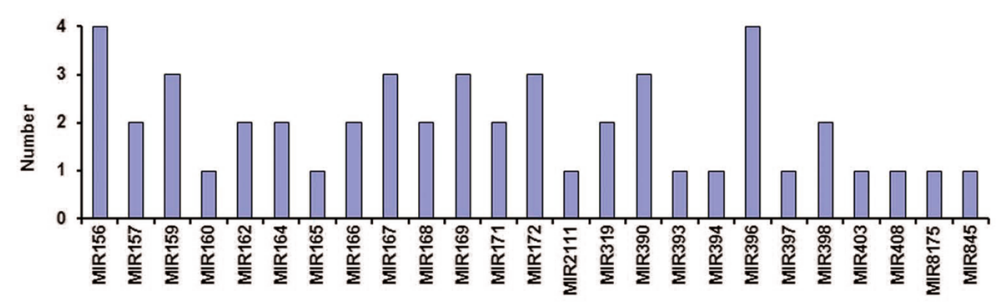

B

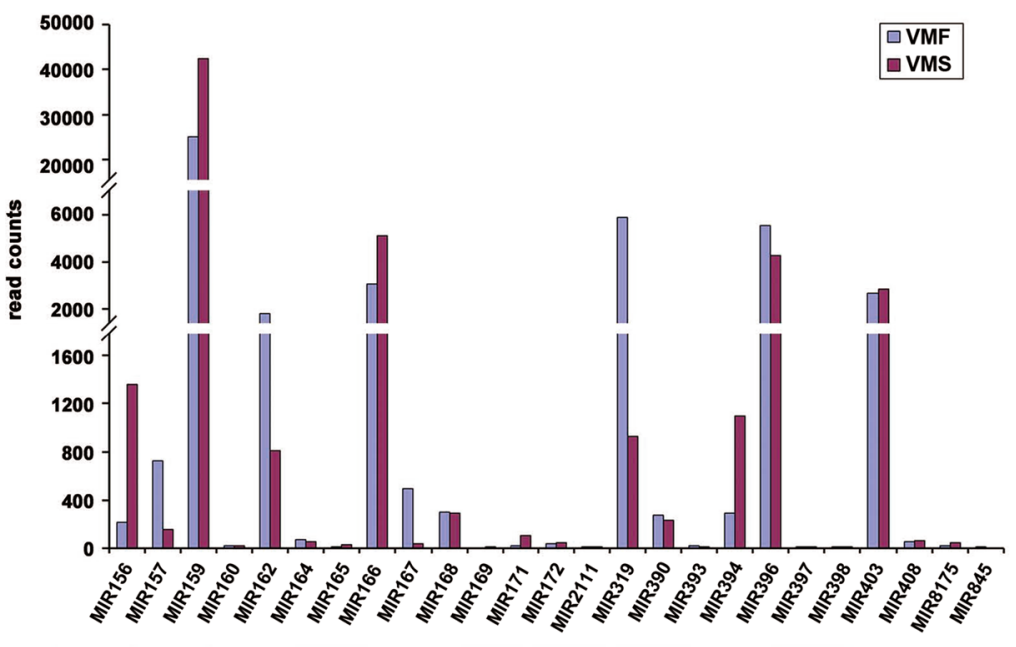

C

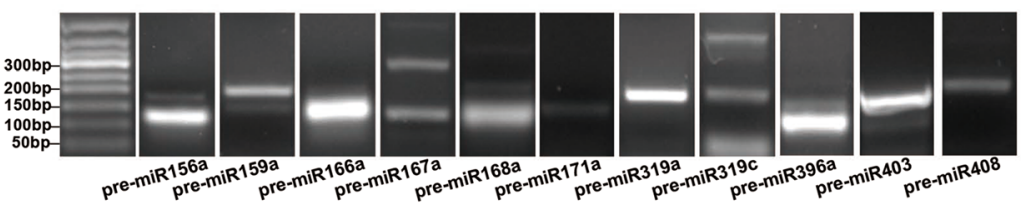

D

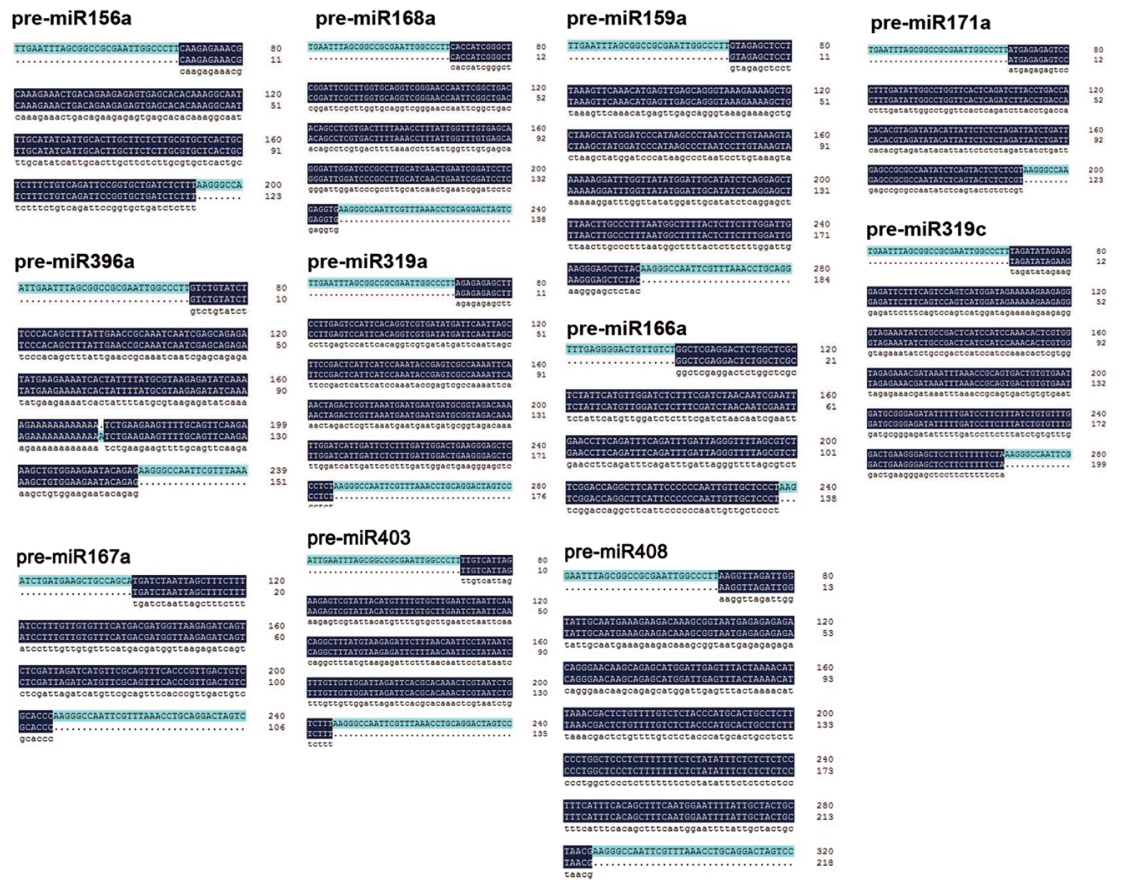

Fig. 4 Known microRNAs (miRNAs) in fertile and sterile flowers. a Numbers of members identified in the 25 conserved miRNA families. b Read counts of each known miRNA family. c Pre-miRNA PCR results of some known miRNAs under UV transillumination. $\mathbf{d}$ Sequence alignment results and Illumina sequencing of known miRNAs 


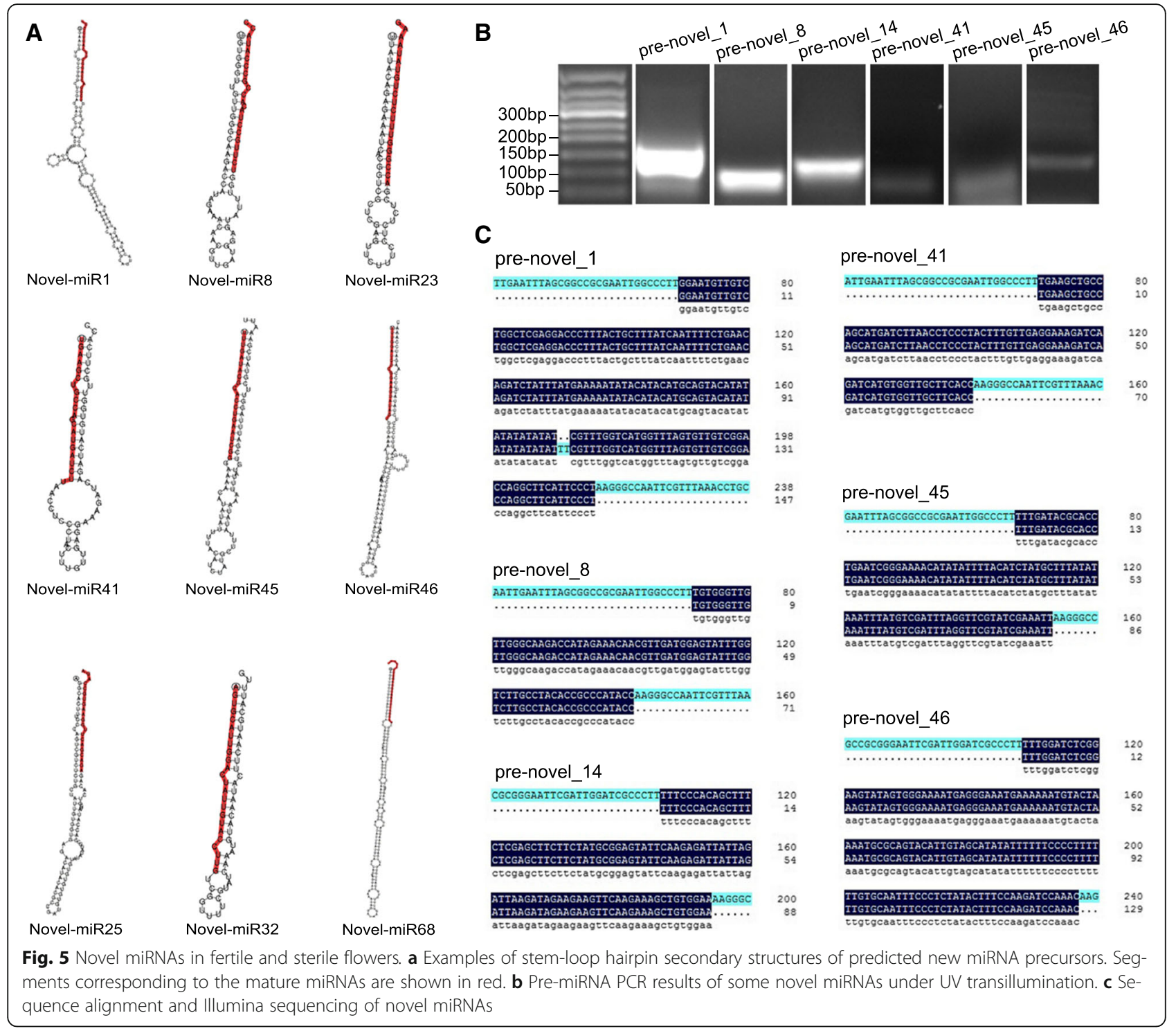

nucleotide bias analysis revealed that $\mathrm{U}$ appeared at the beginning of the miRNAs with a high frequency, 78.34\% on average (Additional file 10). In novel miRNAs, the expression levels of each mature miRNA were obviously different. The expression of novel_1, 10, 4, and 6 were all above 1000 in VMS and VMF, whereas the expression levels of many novel miRNAs (e.g., novel_100, 102, 103, $105,55,57$, and 59) were less than 10 (Additional file 11).

The precursor sequences of the 28 known and novel miRNA candidates were further validated by sub-cloning techniques (PCR amplification and Sanger sequencing). Of the 28 sequences validated by Sanger sequencing, 15 were completely identical to hairpin sequences, whereas 2 had fewer than three mismatched nucleotides (Fig. 4c and Fig. $5 \mathrm{~b})$. Of the known miRNAs, the pre-miRNA sequences of miR156a, miR159a, miR165a, miR167a, miR319a, and miR171a were perfectly matched with the statistics in the library, and miR396a had one mismatched nucleotide (Fig. $4 \mathrm{~d})$. Of the novel miRNAs, the pre-miRNA sequences of novel_8, 14, 41, 45, and 46 were identical to the sequences obtained from sRNA sequencing, and novel_1 contained two mismatched nucleotides (Fig. 5c).

\section{Analysis of miRNAs and their targets in fertile and sterile flowers}

To further understand the functions of known and novel miRNAs, we predicted their putative targets by employing the web-based software psRNATarget and psRobot_tar in psRobot, with default parameters. We found 630 miRNA-target pairs for known miRNAs and 1209 for novel miRNAs (Additional files 12 and 13). The number of predicted targets varied from 1 to 141 per miRNA, and miRNAs of the same family had similar targets. A total of 190 unigene sequences were predicted to be the 
targets of 49 known miRNAs, and 384 unigene sequences were predicted to be the targets of 67 novel miRNAs (Additional files 14 and 15).

These target genes belong to several gene families predicted to play roles in a broad range of physiological processes. Of these targets, nine SBP-box genes were potentially regulated by miR156 and miR157 family members; four MYB genes were potentially regulated by novel_83, novel_87, and novel_112, and one AP2 gene was potentially regulated by miR172 and novel_50, respectively, suggesting complex and specific regulation of flower development by miRNAs. Four and three genes annotated as GRAS and ARF genes, respectively, which are closely associated with plant growth, were identified among the miRNA targets. Other putative gene families identified included NAC, bHLH, and CAMTA. Other than genes known to be members of transcription factor families, many target genes were annotated with their assumed functions associated with cellular components and biological processes. Additionally, a small proportion of the putative target genes had no known functions or significant similarities to other genes in the databases, implying the existence of unknown and specific flower development regulatory pathways.

To investigate the miRNAs involved in flower development, we compared normalized miRNA levels between VMF and VMS (Fig. 6a). miRNAs that had fold change $\log 2>1$ or $<-1$, and $p<0.01$, were considered to be differentially expressed. Among these, 30 known and 39 novel miRNA sequences were differentially expressed (Fig. 6b, Additional file 16). Compared with the comparable data for fertile flowers, in sterile flowers, 14 known miRNAs were upregulated and 16 were downregulated, whereas 21 novel miRNAs were upregulated and 18 were downregulated (Fig. 6c).

Along with the transcriptome, we also constructed a network of miRNAs that regulated mRNA based on differentially expressed miRNA and targets (Fig. 7a). Novel_87 targeted 11 differentially expressed genes, implying that novel miRNAs may play regulatory roles. Additionally, novel_50 and miR172e-5p were found to target the same genes (Fig. 7a).

Furthermore, we compared the expression profiles of the differentially expressed miRNAs and target genes (Additional files 17 and 18). A heat map showed that the expression levels of most of the known and novel miRNAs were negatively correlated with those of their target genes, which is consistent with the gene-silencing function of miRNAs (Fig. 7b). Although the functions of half the novel miRNA targets are unknown, we found that some individual novel miRNAs may target important transcription factors (Fig. 7b; Additional file 19). For example, c50550_g1 was described as $A B C$ transporter $G$ family member 25 , which is involved in the intercellular ABA signaling pathway in Arabidopsis thaliana [32]. Additionally, c43943_g1 was described as GRF4, which plays a role in the regulation of cell expansion in leaf and cotyledon tissues in A. thaliana [33]. However, the expression of only about $20 \%$ of target genes were positively correlated with their corresponding known miRNAs and novel miRNAs enriched in flowers. We cannot rule out the possibility that these miRNAs are not responsible for the differential expression patterns of their target genes, because gene expression can be influenced by many factors. These data indicated the difference between the expression profiles of known and novel miRNAs and their targets in $V$. macrocephalum f. keteleeri flowers.

\section{Expression of miR156 and its targets}

We particularly focused on miR156 family members, based on their expression in the network and the heat map. In the network of miRNA and targets, miR156 family members (miR156a-5p, miR156h, miR156g, and miR156j) with miR157 family members (miR157a-5p, miR157d) regulated similar potential targets (c106171_g1, c107764_g1, c11862_g1, c30688_g1, c32903_g1, c33287_g2, etc.) (Fig. 7a).

In addition, we found the expression of some miR156 family members (miR156a-5p, miR156g, miR156j) to be the opposite of the expression of the SBP family genes (SPL1, SPL5, SPL6, SPL15, SPL16), which is involved in flower transition and late development (Fig. 7b). Based on the expression of 1352 and 213 reads in VMS and VMF, miR156a-5p showed obvious expression differences. miR156g and miR156j were expressed only in VMS. The expression levels of their potential targets SPL1, SPL5, SPL6, SPL15, and SPL16 were greater in VMF than in VMS.

\section{qRT-PCR validation of the miRNAs}

To validate the dynamic expression patterns of the miRNAs, we analyzed the expression patterns of 20 known miRNAs and 15 novel miRNAs with known precursor sequences by qRT-PCR at the MS of flower development (Fig. 8). The relative transcript levels of 16 known miRNAs (Fig. 8a) and 12 novel miRNAs (Fig. 8b) were similar to those observed in the sequencing results (Fig. 8a, b). However, the relative transcript levels of four known miRNAs and three novel miRNAs at this stage were different from those in the sequencing results (Additional file 20). Potential explanations for these inconsistent miRNA expression results include differences in the preparation of the library versus validation samples and the lack of replicates in the sequencing library.

To assess the influence of the miRNAs on their putative targets, we analyzed the expression correlations between miRNAs and their identified targets during three different floral growth stages (Additional file 21). Six interesting miRNA/target modules were identified by qRT-PCR analysis (Fig. 9, Additional file 22). A comparison of the expression levels observed at ES with those at MS revealed negative relationships of miR156a-5p and novel_1 with 


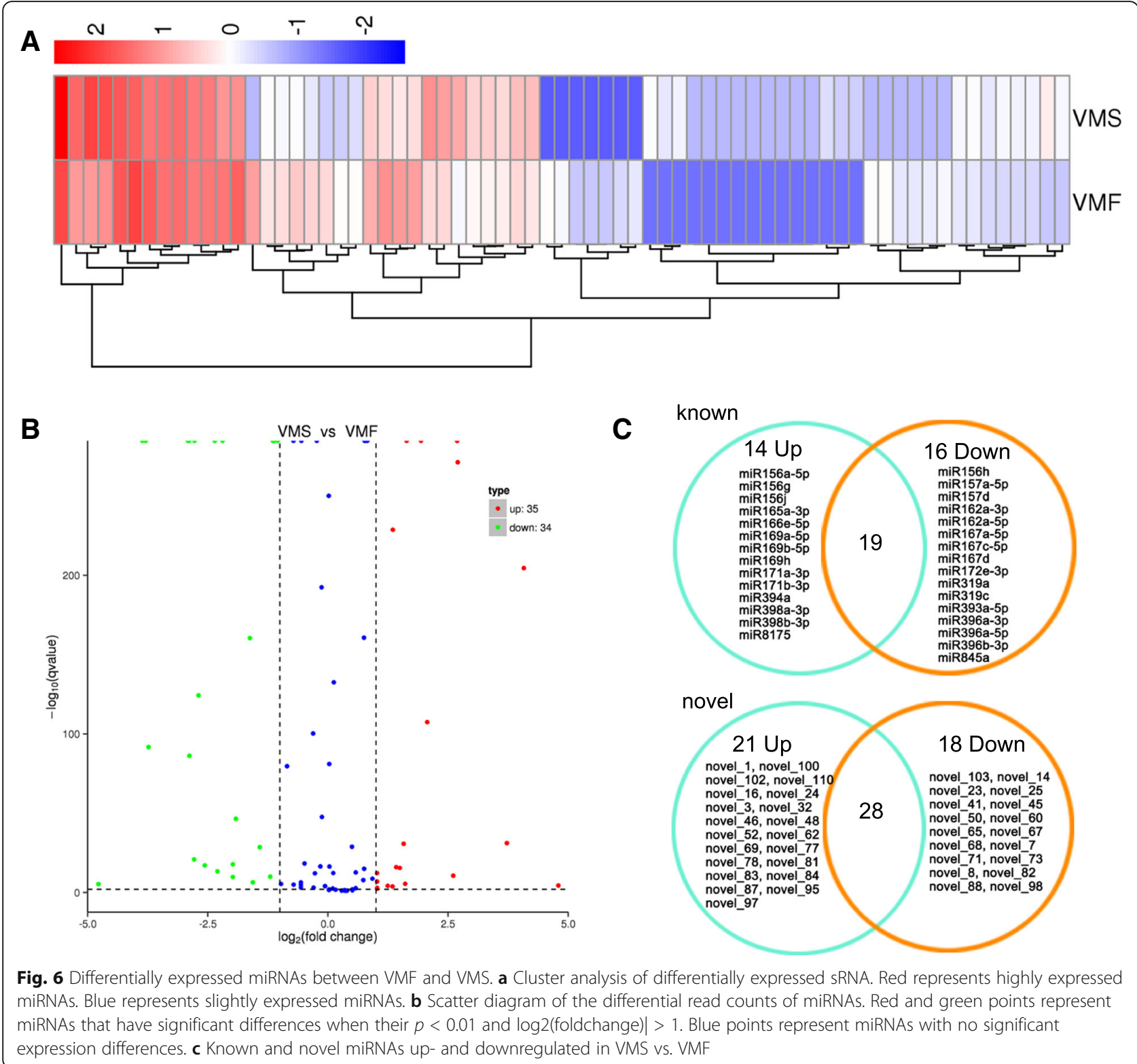

their corresponding gene targets (Fig. 9a, b). The expression level of c47935-g1 gradually increased, while that of the corresponding miR319a decreased, from the ES to LS in VMS (Fig. 9c). In addition, while the expression of miR396b decreased dramatically, that of the target c53763-g1 increased, from the MS to LS in VMF (Fig. 9d).

\section{Discussion}

Complex sRNA populations involved in the differentiation and development of fertile and sterile flowers

During the life cycle of flowering plants, flower development is one of the most important processes leading to the emergence of an organ oriented toward sexual reproduction [34]. To achieve the highest fitness, floral traits are largely determined during adaptive evolution by their capability for sexual reproductive success [35]. Flower size, biomass, and number are variable at the inflorescence and individual levels, as they are considered a consequence of the trade-off rule [36]. Therefore, when plants possess both sterile and fertile flowers, a large size is often associated with sterile or infertile flowers, contributing to diverse flower morphologies and structures, such as in Viburnum (Adoxaceae), Hydrangea (Hydrangeaceae), and Asteraceae. Here, we analyzed the differences between sterile and fertile flowers of $V$. macrocephalum $\mathrm{f}$. keteleeri and found that the size of sterile petals was more than 20 times that of fertile petals in the LS. Furthermore, the stamens of sterile flowers are short with no anthers, and the pistils 
A
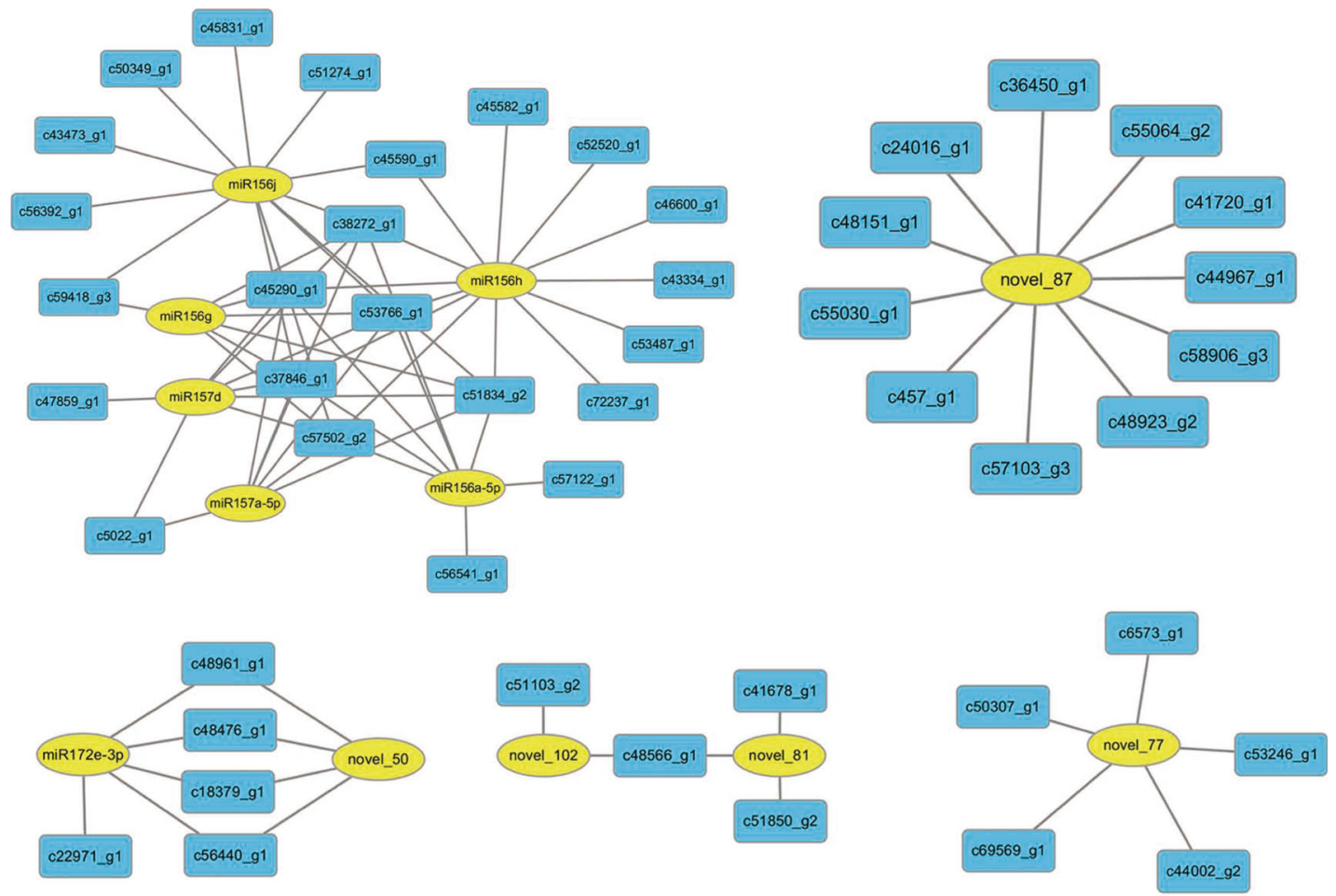

B

miRNA VMS VMF VMS VMF Gene ID Description

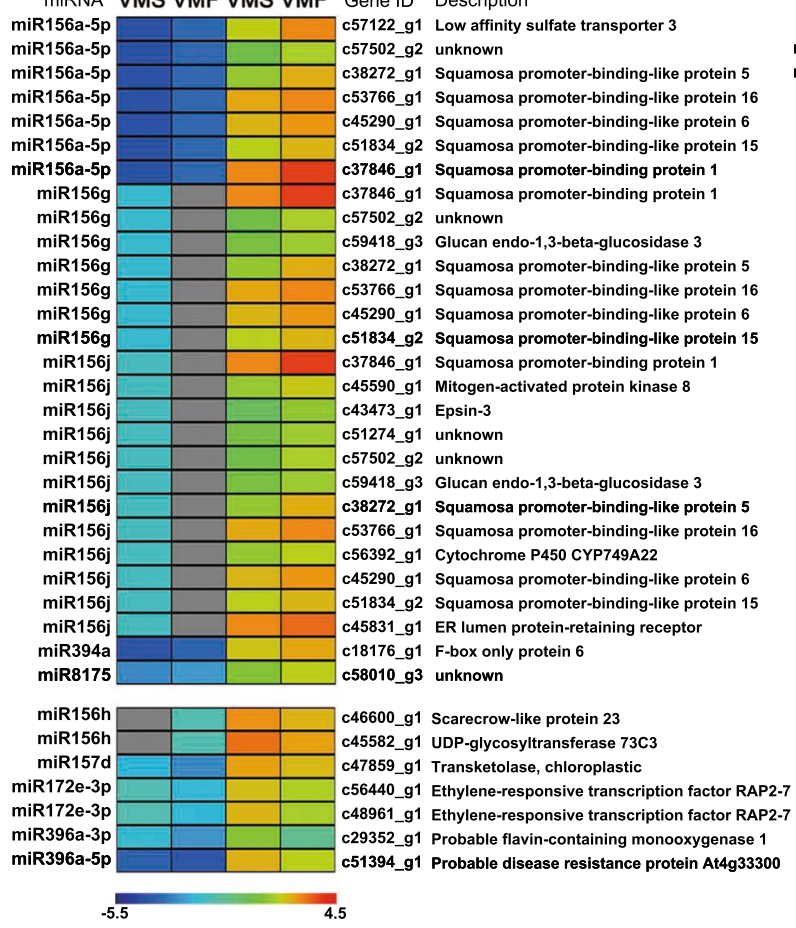

miRNA VMS VMF VMS VMF Gene ID Description

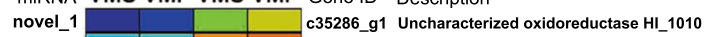

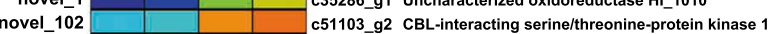
novel_110 - c51296_g1 unknown

novel_16 $\quad$ c50550_91 ABC transporter $\mathrm{G}$ family member 25

novel_24 $\square$ c43943_g1 Growth-regulating factor 4

novel_3 3 c44066_91 Transcription factor GAMYB

novel_32 1 c49447_91 Homeobox-leucine zipper protein ATHB-7

$\begin{aligned} & \text { novel_32 } \\ & \text { novel_32 }\end{aligned}$
c49051_g1 Putative invertase inhibitor

novel_32 13 c39765_91 unknown

novel_46 $1 \quad$ c60274_g1 unknown

novel_48 $\square-1 \quad$ c73673_g1 unknown

novel_77 $\quad$ c50307_91 unknown

novel_77 $\quad$ c6573_g1 unknown

novel_77 $\quad$ c69569 g1 F-box/LRR-repeat protein At4914096

novel_81 $\quad$ c41678_91 unknown

novel_81 10 c51850_92 Protein NRT1/ PTR FAMILY 7.3

novel_83 $\longrightarrow$ c59059_91 unknown

novel_83 $\quad$ c47124_91 Probable xyloglucan hydrolase protein 30

novel_83 10 c45696_91 unknown

novel_87 $\quad \longrightarrow$ c58906_93 unknown

novel_87 $\quad$ c55064_92 unknown

novel_87 $\quad$ c57103 g3 unknown

novel_87 148151 g1 Cysteine-rich receptor-like protein kinase 3

novel_87 $\quad$ c24016_91 Probable WRKY transcription factor 56

novel_87 1 c44967_g1 unknown

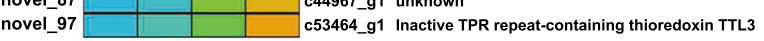

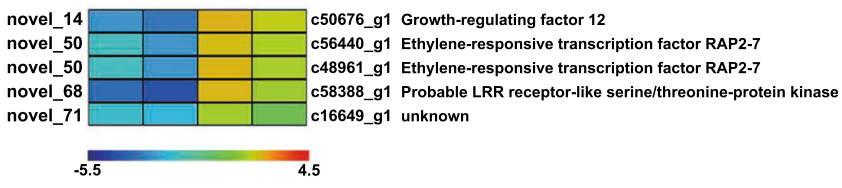

Fig. 7 Network and heat maps of miRNAs and their targets. a Network of miRNAs (yellow) and their targets (blue). $\mathbf{b}$ A combined view of the inverse correlation between the expression of miRNAs and that of their target genes in VMS and VMF. The expression of miRNAs (left) in VMS and VMF was determined by sRNA sequencing. The miRNA targets (right) were predicted by computational methods, and their expression was determined by RNA sequencing. The bar represents the expression scale for the miRNAs (-log10 transcripts per million) and target genes (log 10 transcripts per million) in VMS and VMF. Higher miRNA expression levels are indicated by dark blue and higher target gene expression levels by dark red. Non-expressed miRNAs are shown in grey 


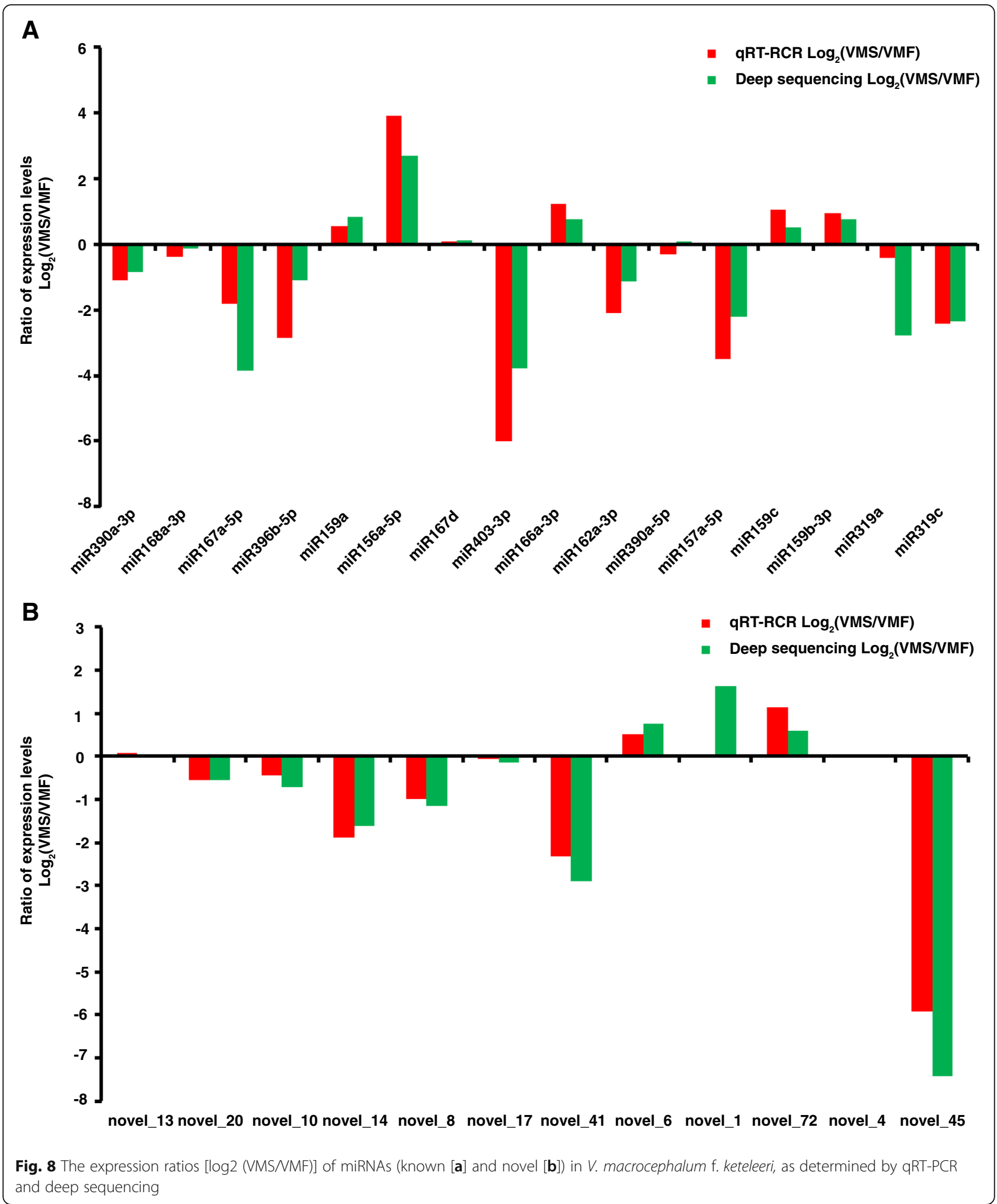

contain abnormal embryo sacs, compared with the normal structures of fertile flowers. These vast phenotypic differences between fertile and sterile flowers from the same genetic background are interesting in terms of attempts to understand their underlying molecular mechanisms.

MiRNAs are important regulators of flower development and floral organ differentiation [37]. Although 

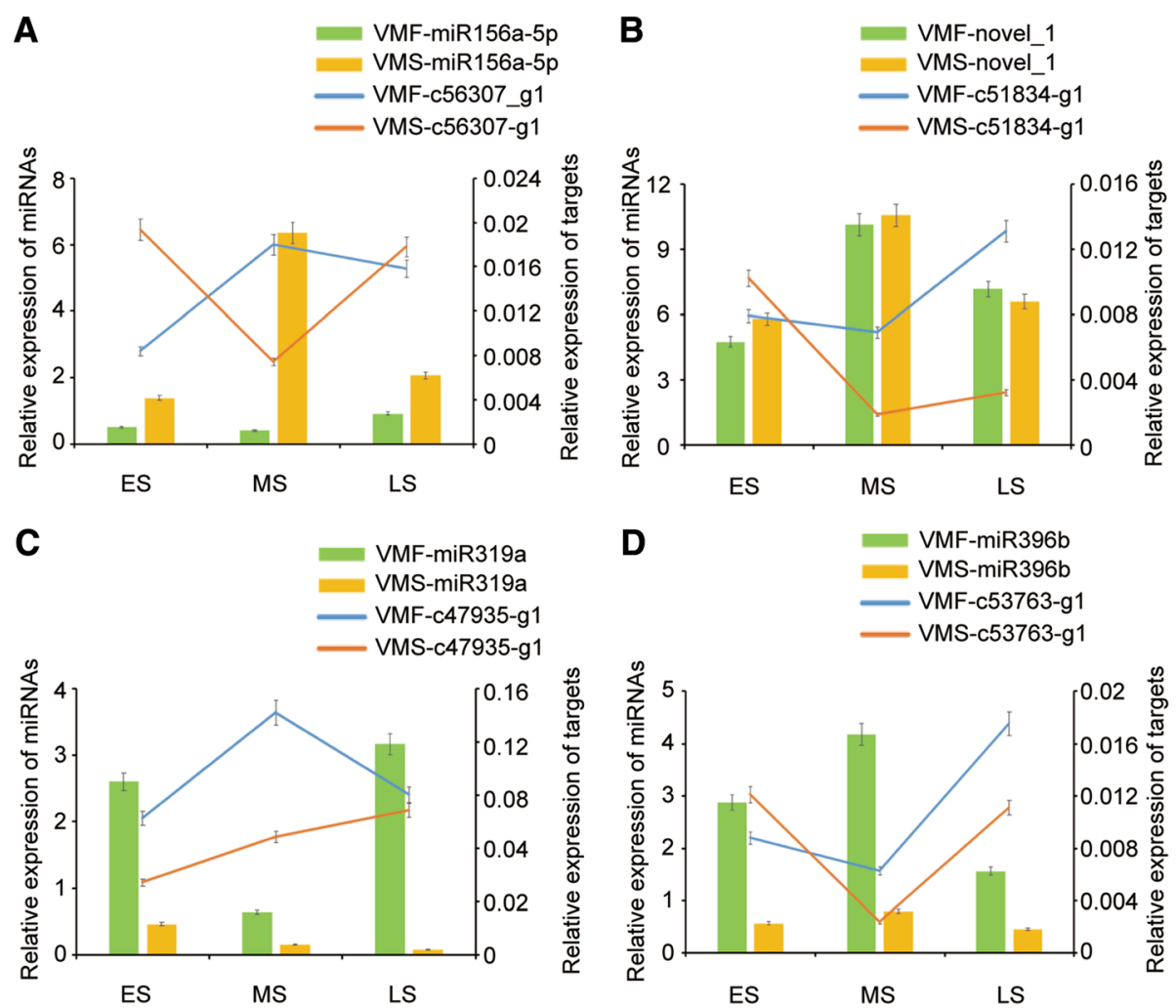

Fig. 9 Correlations between miRNA and target gene expression. a-d The bars and lines indicate the expression levels of miRNAs and their corresponding targets, respectively, at the three different floral growth stages, as determined by qRT-PCR. The left and right y-axes represent the expression levels of the miRNAs and target genes, respectively. The error bars indicate the standard deviations

much effort has been devoted to the cloning and identification of key genes involved in floral development and flowering regulation, the role of miRNAs in floral developmental processes such as flower size and flower organ differentiation is poorly understood [38]. In our study, we constructed sRNA populations of VMF and VMS. The two sRNA libraries both have abundant, highquality data. Known and novel miRNAs were identified, and several of these were verified by sub-cloning techniques. These results suggest that there is a complex and diverse array of sRNAs involved in the development and differentiation of fertile and sterile flowers.

\section{Known miRNAs and their targets associated with the differentiation and development of flowers}

MiRNAs negatively regulate certain genes involved in plant development by directing RNA cleavage or inhibiting translation of target transcripts. Furthermore, molecular and genomic studies in seed plant species have demonstrated that a number of miRNAs and their target genes are involved in the coordinated regulation of flower differentiation and development [39]. For example, miR164 and its targets are thought to be involved in carpel fusion in addition to their roles in sepal and petal boundary development [40]. MiR159 and its targeted GAMYB-related genes are required for normal anther development in Arabidopsis and rice [41-43]. Apart from their functions in the formation of normal flower tissue, which may influence fertility, miRNAs also contribute to flower shape and size. In Arabidopsis, a loss-of-function miR319a mutant exhibited a reduction in petal width and decreased petal and stamen length, supporting the hypothesis that miRNA319 is critical for controlling the size and shape of floral organs by targeting TCP genes [44]. These findings suggest that miRNAs have diverse biological functions in flower organ development and formation.

In this study, a total of 30 known miRNAs were differentially expressed in VMF and VFS. Among them, 14 miRNAs were significantly upregulated in VMS, whereas the other 16 miRNAs were downregulated in VMS. As for the differentially expressed miRNAs, miR156g, miR156j, miR166e-5p, miR169, and miR398a-3p were detected only in VMF, whereas miR156h, miR162a-5, miR167c-5p, and miR845a were found only in VMS. Additionally, some miRNAs had significantly different expression levels. For example, in miR319 family members, the read counts of miR319a were 4164 and 598, whereas those of miR319c were 1725 and 332 in VMF and VMS, respectively. These results suggest that these 
differentially expressed miRNAs might be involved in the development of fertile or sterile flowers.

Our transcriptomic analysis showed that many transcription factors exhibit dynamic gene expression changes in $V$. macrocephalum $\mathrm{f}$. keteleeri flowers [23], and some of these genes are miRNA targets. A total of 190 targets of known miRNAs were predicted and annotated with gene descriptions. For example, miR160 targeted ARF8, one of the ARF family members, which is critical for the proper development of stamens and ovules [45]. Additionally, we found some direct development-related miRNAs involved in plant growth and development. For example, miR159 target genes encode F-box proteins, which play several important roles in flower development [46-48]. Furthermore, some miRNA-targeted transcription factors, such as bHLH and NAC, which are involved in biological synthesis and stress resistance, were found in sterile and fertile flowers [49-51]. We predicted that miR159, miR156, and miR394 regulate genes encoding transcription factors of the bHLH family and that miR164 regulates genes encoding transcription factors of the NAC family. The target genes of flower-related miRNAs suggest that miRNAs specific to flower tissues might have broad regulatory functions with regard to transcription factors, hormones, biosynthesis, and stress responses.

miR156 is widely known for repressing the expression of SPL genes [52]. SPL genes encode plant-specific transcription factors that play important roles in plant phase transition, flower and fruit development, plant architecture, sporogenesis, and response to copper and fungal toxins [53]. In the leaves of Arabidopsis, the effects of both miR156-targeted SPL genes on organ size are correlated with changes in plastochron length, potentially influencing leaf size [54]. In flowers, besides regulating multiple important and divergent biological processes such as flowering time, SPLs also regulate genes mediating cell division, differentiation, and specification in early anther development. Fertile Arabidopsis flowers with secure male fertility require the action of multiple miR156/7-targeted SPL genes in concert with SPL8 [55]. Additionally, an SBP-box transcription factor, tasselsheath4 (tsh4), is a target of miR156 and is known to regulate the development of bracts and meristem boundaries in maize [56]. In our study, four members of the miR156 family were obtained in VMS and VMF, including miR156a-5p, miR156g, miR156h, and miR156j. In addition to miR156h, which was expressed only in VMS, the expression levels of the other three were all significantly higher in VMF than in VMS. Based on the findings that sterile flowers produce few anthers, while fertile flowers produce abundant anthers, miR156 might be involved in the regulation of stamen development. The role of miR156 in flower differentiation and development needs to be further determined using molecular genetics studies.

\section{Targets of the novel miRNA candidates involved in flower development}

The characteristic hairpin structure of miRNA precursors was used to predict novel miRNAs [31]. According to the novel miRNA annotation criteria, we obtained unique sRNA sequences with complementary miRNA*s. Among these miRNAs, several novel miRNAs were significantly differentially expressed in fertile and sterile flowers, including miRNAs found only in VMF/VMS. These results suggest that the novel miRNAs might play a regulatory role in the development and differentiation of flowers of $V$. macrocephalum f. keteleeri.

In terms of the annotation of predicted targets, we found that some novel miRNAs targeted the same transcription factor families as known miRNAs. We predicted that genes encoding bHLH transcription factors are targeted by novel_112, novel_46, novel_96, and novel_62. Additionally, several novel miRNAs (novel_87, 40, 64, 97, 55, and 52) and one known miRNA (miR156) were predicted to target genes encoding cytochrome P450 enzymes, which play important roles in the biosynthesis of flavonoids, including anthocyanins, which contribute to flower color [57]. These results suggest that some novel miRNAs have functions similar to those of known miRNAs in that they target the same transcription factor families or genes. Additionally, we found novel_50 and miR172e_5p targeted the same unigenes. According to a BLAST search with the plant non-coding RNA database website (https://doi.org/10.1093/nar/ gku1162), the mature sequence of novel_50 is consistent with miR172c in Vitis vinifera; thus, we presume that novel_50 in $V$. macrocephalum $\mathrm{f}$. keteleeri may be a candidate member of the miR172 family of important regulatory miRNAs in flowers.

Novel miRNAs have their own targets, and these differ from the targets of known miRNAs. We predicted the identification of two transcription factors involved in hormone regulation in $V$. macrocephalum f. keteleeri flowers. WRKY transcription factors are one of the largest families of transcriptional regulators in plants and form integral parts of signaling webs that modulate many plant processes $[58,59]$. Here, in differentially expressed genes, novel_87 and novel_96 were predicted to target c24016_g1 and c38632_g1, respectively, belonging to the WRKY transcription factor family. The transcription factor LATERAL ORGAN BOUNDARIES (LOB) negatively regulates accumulation of the plant hormone brassinosteroid (BR) at organ boundaries [60]. c27700_g1, targeted by novel_87, was annotated as LOB, and c44637_g1, one target of novel_14 in $V$. macrocephalum f. keteleeri, was annotated as GRF3. 
Additionally, the novel miRNAs function in stress response and biological processes. Furthermore, we predicted c17130_g1, targeted by novel_96, to encode a WUSCHEL-related homeobox member, which plays crucial roles in plant development by regulating cell division and differentiation [61]. These results suggest that some novel miRNAs, including novel_87, novel_96, and novel_14, play important roles in hormone regulation, disease resistance, and biological processes during the differentiation and development of fertile and sterile flowers in $V$. macrocephalum f. keteleeri.

\section{Conclusion}

The fertile and sterile flowers of $V$. macrocephalum $\mathrm{f}$. keteleeri show a distinct disparity in morphology and fertility; these differences are the results of differences in petal cell size, number, and the differentiation of stamens and carpels. Two sRNA libraries were constructed, and miRNAs differentially expressed between fertile and sterile flowers were identified. Some miRNA precursors were validated by sub-cloning, and the dynamic expression levels of miRNAs, such as miR160, miR156, miR164, novel_87, novel_14, and novel_96, and their target genes were determined by qRT-PCR. Our work showed that miRNAs potentially play roles in differentiation and development of fertile and sterile flowers.

\section{Additional files}

Additional file 1: All the primers used in this study, including 29 pairs of primers for precursor sequences, 10 pairs of primers for quantitative real-time PCR of precursor sequences, and 6 pairs of primers for quantitative real-time PCR of target genes. (XLSX $16 \mathrm{~kb}$ )

Additional file 2: Summary of clean 18-30 nt sRNAs. (XLSX $10 \mathrm{~kb}$ )

Additional file 3: Summary of mapped sRNAs. (XLSX $10 \mathrm{~kb}$ )

Additional file 4: Non-coding RNAs among the sRNAs. (XLSX $10 \mathrm{~kb}$ )

Additional file 5: Fold-back structures for known miRNA from Viburnum macrocephalum f. keteleeri. Precursor sequences for known miRNAs were shown in black letters with miRNA sequences highlighted in red. (PDF $1539 \mathrm{~kb})$

Additional file 6: Nucleotide bias analysis of known miRNAs in VMF and VMS. (PDF $231 \mathrm{~kb}$ )

Additional file 7: Known miRNAs in Viburnum macrocephalum $\mathrm{f}$. keteleeri flowers, including family members, mature sequence, length, and expression levels in fertile (VMF) and sterile (VMS) flowers. (XLS $37 \mathrm{~kb}$ )

Additional file 8: Fold-back structures for novel miRNA from Viburnum macrocephalum f. keteleeri. Precursor sequences for novel miRNAs were shown in black letters with miRNA sequences highlighted in red. Precursor secondary structures and MEF value were produced using the MFOLD (http://unafold.rna.albany.edu/?q=mfold/) software. Precursor sequences for novel miRNA*s were shown in black letters with miRNA sequences highlighted in green. (PDF $2121 \mathrm{~kb}$ )

Additional file 9: Novel miRNA*s in Viburnum macrocephalum f. keteleeri flowers, including expression levels in VMF and VMS. (XLS $22 \mathrm{~kb}$ )

Additional file 10: Nucleotide bias analysis of novel miRNAs in VMF and VMS. (PDF $237 \mathrm{~kb}$ )

Additional file 11: Novel miRNAs in Viburnum macrocephalum f. keteleeri flowers, including expression levels in VMF and VMS. (XLSX $11 \mathrm{~kb}$ )
Additional file 12: Known miRNA and predicted targets. (XLSX $19 \mathrm{~kb}$ ) Additional file 13: Novel miRNA and predicted targets. (XLSX 29 kb) Additional file 14: Known miRNA and predicted targets with annotation. (XLSX $24 \mathrm{~kb}$ )

Additional file 15: Novel miRNA and predicted targets with annotation. (XLSX $39 \mathrm{~kb}$ )

Additional file 16: Normalized expression of miRNAs. Log2 Fold_change and $p$-value are obtained for searching differentially expressed miRNAs. (XLSX $13 \mathrm{~kb}$ )

Additional file 17: Up-regulated miRNAs in VMS and related mRNA. (XLSX $10 \mathrm{~kb}$ )

Additional file 18: Down-regulated miRNAs in VMS and related mRNA. (XLSX $10 \mathrm{~kb}$ )

Additional file 19: Differentially expressed genes with gene description. (XLSX $606 \mathrm{~kb}$ )

Additional file 20: miRNAs have opposite tendency between qRT-PCR and sRNA sequencing. (PDF $420 \mathrm{~kb}$ )

Additional file 21: Expression changes of miRNAs at different developmental stages between VMF and VMS. (PDF $2509 \mathrm{~kb}$ )

Additional file 22: Expression changes between miRNAs and targets at different developmental stages. (PDF $1595 \mathrm{~kb}$ )

\section{Abbreviations}

ARF: Auxin response factor; ES: Early stage; GRFS: Growth-regulating factors; LS: Later stage; miRNA: micro RNA; MS: Middle stage; SBP: Squamosa promoter binding protein; SPL: Squamosa promoter binding protein-like; SRNA: small RNA; TCP: Teosinte Branched/Cycloidea/PCF; VMF: Fertile flowers in Viburnum macrocephalum f. keteleeri; VMS: Sterile flowers in Viburnum macrocephalum f. keteleeri

\section{Acknowledgements}

We thank Novogene Company (Beijing) for helping with sequencing and technical assistance.

\section{Funding}

This work was financially supported by the National Natural Science Foundation of China (No. 31670695), Three New Forestry Engineering Foundation of Jiangsu Province (No. LYSX[2016]55). The funding bodies did not have any involvement in the design of the study or in collection, analysis, interpretation of data and paper writing.

\section{Availability of data and materials}

The following link has been created to allow review of record GSE90887: https://www.ncbi.nlm.nih.gov/geo/query/acc.cgi?acc=GSE90887

\section{Authors' contributions}

WXL, LZ and BJ carried out the design of the study and drafted the manuscript. LZ and ZCH performed the experimental work and data analysis. WXL, ZCH, LZ, ZGL, JX, and JWC participated in sample collection, RNA extraction, quantitative RT-PCR and data analysis. WXL, ZCH, LZ, LW and BJ revised the manuscript. All authors read and approved the final manuscript.

\section{Ethics approval and consent to participate}

Viburnum macrocephalum f. keteleeri materials were collected in Yangzhou University, China. This plant species is widespread in eastern China, and it is not classified as an endangered species. Permits are not required for plant material collection. The plant materials are maintained following the institutional guidelines of College of Horticulture and Plant Protection, Yangzhou University, China.

\section{Consent for publication}

Not applicable.

\section{Competing interests}

The authors declare that they have no competing interests. 


\section{Publisher's Note}

Springer Nature remains neutral with regard to jurisdictional claims in published maps and institutional affiliations.

Received: 18 January 2017 Accepted: 5 October 2017

Published online: 13 October 2017

\section{References}

1. Lawton-Rauh AL, Alvarez-Buylla ER, Purugganan MD. Molecular evolution of flower development. Trends Ecol Evol. 2000;15(4):144-9.

2. Tan FC, Swain SM. Genetics of flower initiation and development in annual and perennial plants. Physiol Planta. 2006;128(1):8-17.

3. Lohmann JU, Hong RL, Hobe M, Busch MA, Parcy F, Simon R, Weigel D. A molecular link between stem cell regulation and floral patterning in Arabidopsis. Cell. 2001;105(6):793-803.

4. Cooley AM, Carvallo G, Willis JH. Is floral diversification associated with pollinator divergence? Flower shape, flower colour and pollinator preference in Chilean Mimulus. Ann Bot. 2008;101(5):641-50.

5. Irish VF. The flowering of Arabidopsis flower development. Plant J. 2010; 61(6):1014-28.

6. Clegg MT, Durbin ML. Flower color variation: a model for the experimental study of evolution. Proc Natl Acad Sci U S A. 2000;97(13):7016-23.

7. Galen C. Why do flowers vary? The functional ecology of variation in flower size and form within natural plant populations. Bioscience. 1999:49(8):631-40.

8. Jin B, Wang L, Wang J, Teng N, He XD, Mu XJ, Wang YL. The structure and roles of sterile flowers in Viburnum macrocephalum $\mathrm{f}$. keteleeri (Adoxaceae). Plant Biol. 2010;12(6):853-62.

9. Morales CL, Traveset A, Harder LD. Sterile flowers increase pollinator attraction and promote female success in the Mediterranean herb Leopoldia comosa. Ann Bot 2013;111(1):103-111.

10. Chanderbali AS, Berger BA, Howarth DG, Soltis PS, Soltis DE. Evolving ideas on the origin and evolution of flowers: new perspectives in the genomic era. Genetics. 2016:202(4):1255-65.

11. Kim S, Jin K, Yoo MJ, Kong H, Hu Y, Ma H, Soltis PS, Soltis DE. Expression of floral MADS-box genes in basal angiosperms: implications for the evolution of floral regulators. Plant J. 2005;43(5):724-44.

12. Rhee SJ, Seo M, Jang YJ, Ko YJ, Sim TY, Lee GP. Transcriptome profiling of differentially expressed genes in floral buds and flowers of male sterile and fertile lines in watermelon. BMC Genomics. 2015;16:914

13. Krizek BA, Anderson JT. Control of flower size. J Exp Bot. 2013;64(6):1427-37.

14. Bartel DP. MicroRNA target recognition and regulatory functions. Cell. 2009; 136(2):215-33.

15. Hong $Y$, Jackson S. Floral induction and flower formation - the role and potential applications of miRNAs. Plant Biotechnol J. 2015;13(3):282-92.

16. Nag A, Jack T. Chapter twelve-sculpting the flower; the role of microRNAs in flower development. Curr Top Dev Biol. 2010;91:349-78.

17. Luo Y, Guo Z, Li L. Evolutionary conservation of microRNA regulatory programs in plant flower development. Dev Biol. 2013;380(2):133-44.

18. Nag A, King S, Jack T. miR319a targeting of TCP4 is critical for petal growth and development in Arabidopsis. Proc Natl Acad Sci U S A. 2009;106(52):22534-9.

19. Murray F, Kalla R, Jacobsen J, Gubler F. A role for HvGAMYB in anther development. Plant J. 2003;33(3):481-91.

20. Lu Y, Zhang L, Cheng FM, Zhao JG, Cui JW, Li WX, Wang L, Jin B. The morphology, ultrastructure, element distribution and motion behaviour in pollen of Ginkgo biloba L. Trees. 2016;30:2189-201.

21. Wang L, Lu ZG, Li WX, Xu J, Luo KG, Lu WC, Zhang L, Jin B. Global comparative analysis of expressed genes in ovules and leaves of Ginkgo biloba L. Tree Genet Genom. 2016:12:29.

22. Langmead B, Trapnell C, Pop M, Salzberg SL. Ultrafast and memory-efficient alignment of short DNA sequences to the human genome. Genome Biol. 2009;10(3):R25.

23. Lu ZG, Xu J, Li WX, Zhang L, Cui JW, He QS, Wang L, Jin B. Transcriptomic analysis reveals mechanisms of sterile and fertile flower differentiation and development in Viburnum macrocephalum f. keteleeri. Front Plant Sci. 2017;8:261.

24. Wen $M$, Shen $Y$, Shi S, Tang T. miREvo: an integrative microRNA evolutionary analysis platform for next-generation sequencing experiments. BMC Bioinformatics. 2012;13:140.

25. Friedländer MR, Mackowiak SD, Li N, Chen W, Rajewsky N. miRDeep2 accurately identifies known and hundreds of novel microRNA genes in seven animal clades. Nucleic Acids Res. 2012;40(1):37-52.
26. Wang L, Feng Z, Wang X, Wang X, Zhang X. DEGseq: an $R$ package for identifying differentially expressed genes from RNA-seq data. Bioinformatics. 2010;26:136-8.

27. Wu HJ, Ma YK, Chen T, Wang M, Wang XJ. PsRobot: a web-based plant small RNA meta-analysis toolbox. Nucleic Acids Res. 2012;40:22-8.

28. Srivastava PK, Moturu TR, Pandey P, Baldwin IT, Pandey SP. A comparison of performance of plant miRNA target prediction tools and the characterization of features for genome-wide target prediction. BMC Genomics. 2014;15:348.

29. Turner M, Adhikari S, Subramanian S. Optimizing stem-loop qPCR assays through multiplexed CDNA synthesis of U6 and miRNAs. Plant Signal Behav. 2013;8(8):e24918.

30. Livak KJ, Schmittgen TD. Analysis of relative gene expression data using realtime quantitative PCR and the $2^{-\Delta \Lambda C T}$ method. Methods. 2001;25(4):402-8.

31. Meyers BC, Axtell MJ, Bartel B, Bartel DP, Baulcombe D, Bowman JL, et al. Criteria for annotation of plant microRNAs. Plant Cell. 2008;20(12):3186-90.

32. Kuromoria T, Miyajib T, Yabuuchic H, Shimizud H, Sugimotoa E, Kamiyaa A, Moriyamab Y, Shinozakia K. ABC transporter AtABCG25 is involved in abscisic acid transport and responses. Proc Natl Acad Sci U S A. 2010;107(5):2361-6.

33. Kim JH, Lee BH. GROWTH-REGULATING FACTOR4, of Arabidopsis thaliana, is required for development of leaves, cotyledons, and shoot apical meristem. J Plant Biol. 2006:49(6):463-8.

34. Blázquez MA, Weigel D. Integration of floral inductive signals in Arabidopsis. Nature. 2000:404(6780):889-92.

35. Harder LD, Barrett SCH. Ecology and evolution of flowers. Oxford: Oxford University Press; 2006

36. Sargent RD, Goodwillie C, Kalisz S, Ree RH. Phylogenetic evidence for a flower size and number trade-off. Am J Bot. 2007;94(12):2059-62.

37. Cartolano M, Castillo R, Efremova N, Kuckenberg M, Zethof J, Gerats T, et al. A conserved microRNA module exerts homeotic control over Petunia hybrida and Antirrhinum majus floral organ identity. Nat Genet. 2007:39(7):901-5.

38. David BL, Hernandez K, Taylor SH, Meyer E, Logan TL, Barry KW, et al. The genetics of divergence and reproductive isolation between ecotypes of Panicum hallii. New Phytol. 2015;205(1):402-14.

39. Wollmann H, Weigel D. Small RNAs in flower development. Eur J Cell Biol. 2010;89(2-3):250-7.

40. Larue $C T$, Wen J, Walker JC. A microRNA-transcription factor module regulates lateral organ size and patterning in Arabidopsis. Plant J. 2009;58:450-63.

41. Achard P, Herr A, Baulcombe CD, Harberd PN. Modulation of floral development by a gibberellin-regulated microRNA. Development. 2004;131(14):3357-65.

42. Millar AA, Gubler F. The Arabidopsis GAMYB-Like genes, MYB33 and MYB65, are microRNA-regulated genes that redundantly facilitate anther development. Plant Cell. 2005;17(3):705-21.

43. Aya K, Matsuoka M. Gibberellin modulates anther development in rice via the transcriptional regulation of GAMYB. Plant Cell. 2009;21(5):1453-72.

44. Wu MF, Tian Q, Reed JW. Arabidopsis microRNA167 controls patterns of ARF6 and ARF8 expression, and regulates both female and male reproduction. Development. 2006:133(21):4211-8.

45. Nagpal P, Ellis CM, Weber H, Ploense SE, Barkawi LS, Guilfoyle TJ, et al. Auxin response factors ARF6 and ARF8 promote jasmonic acid production and flower maturation. Development. 2005;132(18):4107-18.

46. Souer E, Rebocho AM, Kusters E, de Bruin RA, Koes R. Patterning of inflorescences and flowers by the F-box protein double top and the leafy homolog aberrant leaf and flower of petunia. Plant Cell. 2008;20(8):2033-48.

47. Dharmasiri N, Dharmasiri S, Estelle M. The F-box protein TIR1 is an auxin receptor. Nature. 2005:435(7041):446-51.

48. Dharmasiri N, Dharmasiri S, Weijers D, Lechner E, Yamada M, Hobbie L, et al. Plant development is regulated by a family of auxin receptor $\mathrm{F}$ box proteins. Dev Cell. 2005;9(1):109-19.

49. Feller A, Machemer K, Braun EL, Grotewold E. Evolutionary and comparative analysis of MYB and bHLH plant transcription factors. Plant J. 2011;66(1):94-116.

50. Olsen AN, Ernst HA, Leggio LL, Skriver K. NAC transcription factors: structurally distinct, functionally diverse. Trends Plant Sci. 2005;10(2):79-87.

51. Zhao J, He Q, Chen G, Wang L, Jin B. Regulation of non-coding RNAs in heat stress responses of plants. Front Plant Sci. 2016;7:1213

52. Wu G, Park MY, Conway SR, Wang M, Wang XJ. The sequential action of miR156 and miR172 regulates developmental timing in Arabidopsis. Cell. 2009:138(4):750-9.

53. Chen X, Zhang Z, Liu D, Kai Z, Li A, Long M. SQUAMOSA promoter-binding protein-like transcription factors: star players for plant growth and development. J Integr Plant Biol. 2010;52(11):946-51. 
54. Wang J, Schwab R, Czech B, Mica E, Weigel D. Dual effects of miR156targeted SPL genes and CYP78A5/KLUH on plastochron length and organ size in Arabidopsis thaliana. Plant Cell. 2008;20:1231-43.

55. Xing SP, Salinas M, Höhmann S, Berndtgen R, Huijser P. miR156-targeted and nontargeted SBP-box transcription factors act in concert to secure male fertility in Arabidopsis. Plant Cell. 2010;22(12):3935-50

56. Chuck $\mathrm{G}$, Bortiri E. The unique relationship between tsh4 and ra2 in patterning floral phytomers. Plant Signal Behav. 2010;5(8):979-81.

57. Tanaka Y, Brugliera F. Flower colour and cytochromes P450. Phytochem Rev. 2006;368(2):283-91.

58. Rushton PJ, Somssich IE, Ringler P, Lin J. WRKY transcription factors. Trends Plant Sci. 2014;15(5):247-58.

59. Rushton DL, Tripathi P, Rabara RC, Lin J, Ringler P, Boken AK, et al. WRKY transcription factors: key components in abscisic acid signalling. Plant Biotechnol J. 2012:10(1):2-11.

60. Bell EM, Springer PS. Arabidopsis lateral organ boundaries negatively regulates brassinosteroid accumulation to limit growth in organ boundaries Proc Natl Acad Sci U S A. 2012;109(51):21146-51.

61. Laux T, Mayer KF, Berger J, Jürgens G. The WUSCHEL gene is required for shoot and floral meristem integrity in Arabidopsis. Development. 1996; 122(1):87-96.

\section{Submit your next manuscript to BioMed Central} and we will help you at every step:

- We accept pre-submission inquiries

- Our selector tool helps you to find the most relevant journal

- We provide round the clock customer support

- Convenient online submission

- Thorough peer review

- Inclusion in PubMed and all major indexing services

- Maximum visibility for your research

Submit your manuscript at www.biomedcentral.com/submit 